\title{
Long noncoding RNA LINC00314 facilitates osteogenic differentiation of adipose- derived stem cells through the hsa-miR- 129-5p/GRM5 axis via the Wnt signaling pathway
}

Zheng-liang Shi, Hua Zhang*, Zhi-yong Fan, Wei Ma, Yong-zhou Song, Ming Li, Tong-qiu Li, Shu-xing Cao and Guo-jun Feng

\begin{abstract}
Background: Many studies have shown that long noncoding RNAs (IncRNAs) are closely related to the stimulation of osteogenic differentiation of adipose-derived stem cells (ADSCs) and the prevention of osteoporosis. Current research aimed to investigate the novel IncRNA and explored the function and molecular mechanism of the LINC00314/miR-129-5p/GRM5 axis in regulating osteogenic differentiation of ADSCs.

Methods: LncRNA and miRNA sequencing was performed in normal and osteogenic differentiation-induced ADSCs (osteogenic group). Abnormally expressed IncRNAs and miRNAs were obtained by the R software and the relative expression of LINC00314, miR-129-5p, and GRM5 during osteogenic induction was measured by RT-PCR. ADSCs were then transfected with pcDNA3.1-sh-LINC00314 and agomiR-129-5p. Alizarin red staining (ARS) and alkaline phosphatase (ALP) staining were performed to identify the mechanism of the LINC00314/miR-129-5p/GRM5 axis in regulating osteogenic differentiation of ADSCS.
\end{abstract}

Results: LINC00314 was significantly upregulated in the group of osteogenic-induced ADSCs. LINC00314 and GRM5 mimics increased the early and late markers of osteogenic differentiation, which manifest in not only the markedly increased ALP activity but also higher calcium deposition, while miR-129-5p mimic had the opposite effects. LINC00314 directly targeted miR-129-5p through luciferase reporter assay, and miR-129-5p suppressed GRM5 expression. Moreover, the LINC00314/miR-129-5p/GRM5 regulatory axis activated the Wnt/ $\beta$-catenin signaling pathway.

Conclusions: LINC00314 confers contributory function in the osteogenic differentiation of ADSCS and thus the LINC00314/miR-129-5p/GRM5 axis may be a novel mechanism for osteogenic-related disease.

Keywords: LINC00314, miR-129-5p, GRM5, ADSCs, Osteogenic differentiation

* Correspondence: fanqinkao19600913@126.com

Department of Orthopedics, The Second Hospital of Hebei Medical

University, No. 215, Hepingxi Road, Shijiazhuang 050000, Hebei Province,

China

(c) The Author(s). 2020 Open Access This article is licensed under a Creative Commons Attribution 4.0 International License, which permits use, sharing, adaptation, distribution and reproduction in any medium or format, as long as you give appropriate credit to the original author(s) and the source, provide a link to the Creative Commons licence, and indicate if changes were made. The images or other third party material in this article are included in the article's Creative Commons licence, unless indicated otherwise in a credit line to the material. If material is not included in the article's Creative Commons licence and your intended use is not permitted by statutory regulation or exceeds the permitted use, you will need to obtain permission directly from the copyright holder. To view a copy of this licence, visit http://creativecommons.org/licenses/by/4.0/. The Creative Commons Public Domain Dedication waiver (http://creativecommons.org/publicdomain/zero/1.0/) applies to the data made available in this article, unless otherwise stated in a credit line to the data. 


\section{Background}

Osteoporosis is a serious degenerative disease characterized by the reduction of bone mass and destruction of the bone microstructure $[1,2]$. Osteoporosis can result in fractures and may increase economic cost and societal burden [3]. Previously, bone marrow mesenchymal stem cells (BMSCs) were administered as seed cells to improve BMD and used for tissue engineering [4]. However, the proliferation ability of BMSCs was limited, especially in older patients. Adipose-derived stem cells (ADSCs) could be a potential alternative for BMSCs due to their advantages of being easy to obtain and widely sourced $[5,6]$.

Long noncoding RNAs (lncRNAs) belongs to a family of small RNAs, which are often 200 or more nucleotides long and play crucial roles in bone disease [7]. Zhang et al. [8] reported that IncRNA NKILA indirectly regulates RXFP1/AKT signaling pathway to regulate osteogenesis. Another study also found lncRNA HOTAIRM1 directly targeting with JNK/AP-1 signaling pathway to control the osteogenic differentiation [9]. Wu et al. [10] analyzed the differentially expressed long noncoding RNAs in the normal and induced groups and found that HIF1A-AS2 has a positive role in promoting ADSCs osteogenic differentiation through the PI3K/Akt signaling pathway. LncRNA LINC00314 is located on chromosome 21 with open reading frame 94. Currently, no study has explored the role and mechanism of LINC00314 in the regulation of ADSC osteogenic differentiation.

There is now overwhelming evidence that alterations in microRNA (miRNA) expression levels are linked to osteogenic differentiation [11]. Several miRNAs have been identified to have potential roles in promoting or inhibiting the osteogenic differentiation of ADSCs [12]. Li et al. [12] found that miR-154-5p could directly bind to the 3 '-UTR of Wnt11 and thus inhibit the osteogenic differentiation. Liu et al. [11] revealed that miR-145-5p suppresses osteogenic differentiation of ADSCs by targeting semaphorin $3 \mathrm{~A}$.

MiR-129-5p participates in regulating the proliferation, migration, and invasion of retinoblastoma cells and affects spinal cord injury $[13,14]$. In a study on the regulation of osteogenic differentiation, Valenti et al. [15] found that physical exercise modulates miR-129-5p expression in progenitor cells and thus promotes osteogenesis. Further study also revealed that miR-129-5p could regulate osteoblast differentiation of BMSCs.

Metabotropic glutamate receptor 5, named GRM5, has been shown to activate phospholipase $C$ and participate in multiple biological processes [16]. Many studies have shown the Wnt/ $\beta$-catenin signaling pathway participant into the osteogenic differentiation of
ADSCs. However, whether LINC00314 could activate the Wnt/ $\beta$-catenin signaling pathway and stimulate osteogenic differentiation of ADSCs remains unclear.

In this study, LINC00314 was found to be abnormally expressed during the osteogenic differentiation of ADSCs through gene chip and further confirmed by qRT-PCR. Further bioinformatics analysis revealed LINC00314 directly regulating with miR-129-5p and its targeting gene GRM5.

\section{Methods}

\section{Tissue specimens and ADSC cultures}

Human adipose tissue was collected from patients who prepared for total knee arthroplasty. Each patient signed an informed consent form, and this research was approved by the ethics committee of the Second Hospital of Hebei Medical University. Collected adipose tissue was washed with PBS three times and cut into sections. Then, these sections were digested with $0.1 \%$ type I collagenase (Gibco, Grand Island, NY, USA) at $37^{\circ} \mathrm{C}$ for $1 \mathrm{~h}$. Next, we added $5 \mathrm{~mL}$ DMEM containing 10\% fetal bovine serum (FBS, Thermo Fisher Scientific, Inc., USA) to inhibit the type I collagenase activity. The cell suspension was centrifuged at $300 \times g$ to collect the cells. Cells were cultured in highglucose Dulbecco's modified Eagle medium (DMEM, Gibco, Grand Island, NY, USA) with 20\% FBS and 1\% penicillin at $5 \% \mathrm{CO}_{2}$ and $37^{\circ} \mathrm{C}$. ADSCs at passage 3 were used for subsequent studies. A previous study revealed that less than $5 \%$ of the ADSCs showed senescence when expanded to generation 10 [17].

\section{ADSCs identification}

To identify ADSC surface markers, ADSCs at passage 3 were collected in tubes at $4 \times 10^{5} /$ tube. Next, anti-CD31PE, anti-CD45-PE, anti-CD44-FITC, anti-CD29-FITC, anti-CD73-PE, anti-CD90-FITC, and anti-CD105-PE (all from BD Biosciences, San Jose, CA, USA) antibodies were incubated. Then, ADSCs in solution were identified by a FACS Calibur flow cytometer (Becton-Dickinson, Franklin Lakes, NJ, USA).

Trilineage differentiation was performed to show the differentiation potency of ADSCs. In brief, ADSCs were seeded into 6 -well plates $\left(5.0 \times 10^{5}\right.$ cells/well $)$ with normal medium until cell confluence reached approximately $70 \%$. The nonadherent cells were removed by replacing the medium, and the attached cells were cultured until confluence. The cells were then grown for 21 days in the adipogenic, osteogenic, and chondrogenic medium (Cyagen, Guangzhou, China). Alizarin red S staining (ARS, Solarbio, Beijing, China) was performed to assess osteogenic differentiation. Adipogenic differentiation was visualized using Oil Red O (Sigma-Aldrich, St. Louis, MO, USA) 
staining. Alcian Blue (Sigma-Aldrich, St. Louis, MO, USA) staining was used to assess chondrogenic differentiation.

\section{Microarray}

Microarray analyses of lncRNA and microRNA expression were performed as described previously [18]. Briefly, total RNA from the normal and induced groups was extracted by TRIzol as described previously. cDNA was synthesized, labeled with fluorescent dye, and hybridized with a lncRNA Human Gene Expression Microarray v4.0 $(4 \times 180 \mathrm{~K}$; Cloud-Seq Biotech, Shanghai, China) platform (LC Sciences, Houston, TX, USA) for IncRNA and microRNA, respectively. Differentially expressed lncRNAs and mRNAs were obtained by the limma package. Moreover, heatmaps and volcano plots were generated by using Bioconductor (http://www.bioiconductor.org) in $\mathrm{R}$ software (Free Software Foundation Inc., Boston, MA, USA).

\section{Bioinformatic analysis}

First, the limma package was utilized to identify differentially expressed lncRNAs and miRNAs in the induced and non-induced groups using the screening criteria $\mid \operatorname{logFC}$ (foldchange) $\mid \geq 1$ and adjusted $P<0.05$. Cluster enrichment of gene ontology (GO) and Kyoto Encyclopedia of Genes and Genomes (KEGG) pathway was performed using the Database for Annotation, Visualization and Integrated Discovery (DAVID) database (https://david.ncifcrf.gov/).

The GO terms including three categories: biological process (BP), cellular component (CC), and molecular function (MF). Target genes network were constructed by the Search Tool for the Retrieval of Interacting Genes (STRING) database.

The interaction of miRNAs with LINC00314 was predicted from a reliable online miRNA reference database, miRcode (http://www.mircode.org/). The prediction of target mRNAs of miRNAs was performed using three databases, miRDB (http://www. mirdb.org/), miRTarBase (http://mirtarbase.mbc.nctu. edu.tw), and TargetScan (http://www.targetscan.org) and the miRNA profile. The ceRNA network was visualized by Cytoscape (https://cytoscape.org/).

\section{Cell transfection}

LINC00314, sh-LINC00314, agomiR-129-5p, pcDNA3.1LINC00314, antagomir-129-5p, pcDNA3.1-GRM5, and their respective negative control (NC) came from GenePharma (Shanghai, China). In brief, lipofectamine 3000 (Invitrogen, Carlsbad, CA, USA) and transfection object were used to transfect for $10 \mathrm{~min}$. The transfection rate of each reagent was confirmed to be $\geq 90 \%$. To inhibit the effects of WNT activation, XAV939 (MCE, Shanghai, China) was utilized to pre-treat ADSCs before pcDNA3.1-GRM5 treatment. XAV939 was used to treat ADSCs at a final concentration of $10 \mu \mathrm{M}$ according to a previous study [19].

\section{qRT-PCR}

Trizol reagent (Takara Bio Inc., Otsu, Shiga, Japan) was applied for total RNA extraction from cells. Afterwards, mRNA levels were determined using the SYBR Green qPCR Mix Kit on the ABI 7500 Fast Real-Time PCR System (Thermo Fisher Scientific Inc., Waltham, MA, USA). All primers were purchased from Invitrogen (Shanghai, China). Glyceraldehyde-3phosphate dehydrogenase (GAPDH) and U6 were utilized for internal control as appropriate. Sequences of primers are presented in Table 1 . The relative quantitative expression of interest genes was expressed as fold change (2- $\Delta \Delta \mathrm{Ct}$ method). $\Delta \Delta \mathrm{Ct}=[\mathrm{Ct}$ (target gene) $\mathrm{Ct}$ (GAPDH/U6)] experimental group - [Ct (target gene) - Ct (GAPDH/U6)] control group. Each sample was examined in triplicate.

\section{Western blot analysis}

The expression level of osteogenic differentiationrelated genes was determined by Western blot analysis. The equivalent cracking solution was added to phenylmethyl sulfonylfluoride until the concentration was $1 \mathrm{mM}$ (No. ST506, Beyotime Biotechnology Co.,

Table 1 Primer sequences for qRT-PCR

\begin{tabular}{lll}
\hline Gene & Forward primer 5'-3' $^{\prime}$ & Reverse primer 5'-3' \\
\hline GAPDH & GACAGTCAGCCGCATCTTCT & GCGCCCAATACGACCAAATC \\
MiR-129-5p & GGCTITTGCGGTCTGG & CAGTGCGTGTCGTGGAGT \\
RUNX2 & CCTTCAAGGTGGTAGCCCTC & CCCTAAATCACTGAGGCGGT \\
Osterix & AGACCTCCAGAGAGGAGAGAC & GGGGACTGGAGCCATAGTGA \\
Osteocalcin & AATAGCCCTGGCAGATTCCC & CTCTCATGGTGTCTCGGTGG \\
LINC00314 & GATCTATTGTTAGCCAT & ATGGCUAAACAAUAGAUC \\
GRM5 & AGATCTGTGGCTCAGTTCCTTT & AATCACTATGAATCCCTGCACCT \\
\hline
\end{tabular}


Table 2 Antibody sources and dilution rate

\begin{tabular}{llll}
\hline Protein name & Company & Cat. No. & Working dilution \\
\hline RUNX2 & Abcam & ab76956 & $1: 1000$ \\
Osterix & Abcam & ab22552 & $1: 3000$ \\
Osteocalcin & Abcam & ab13418 & $1: 2000$ \\
Wnt5a & Proteintech & 55184 & $1: 5000$ \\
$\beta$-catenin & Proteintech & 17565 & $1: 2000$ \\
p- $\beta$-catenin & Abcam & ab73153 & $1: 3000$ \\
GAPDH & Proteintech & 60004 & $1: 5000$ \\
\hline
\end{tabular}

Ltd., Shanghai, China). The transfected cells were lysed with Western and IP cell lysates. After full cracking, the cells were centrifuged at 10000-14000 $\times g$ for 3-5 min with the supernatant collected. Bicinchoninic acid kit (No. p0009, Beyotime Biotechnology Co., Ltd., Shanghai, China) was utilized to determine the protein concentration. Electrophoresis was then performed in polyacrylamide gel $(5 \%$ concentrate and $12 \%$ separation gel). Tris-buffered saline Tween-20 (TBST) containing $5 \%$ bovine serum albumin (BSA) was used to seal the membrane for $1 \mathrm{~h}$ in a decolorizing shaker at room temperature. The sealing solution was discarded, and the membrane was put into the plastic groove added with 5\% BSA to prepare the primary antibody solution of corresponding concentration overnight at $4{ }^{\circ} \mathrm{C}$. The primary antibodies used are shown in Table 2. The membrane was washed with TBST three times, $10 \mathrm{~min}$ each time. Then, the membrane was incubated at $4{ }^{\circ} \mathrm{C}$ for $4 \mathrm{~h}$ with the secondary antibody solution. The membrane was washed with TBST 3 times, 10 min each time. The membrane was immersed in electrochemiluminescence developer (wbkls0100, Merck Millipore, Billerica, MA, USA) for visualization. The relative optical density (OD) of all immunoblotting bands was analyzed. Western blots were quantified with Image J software $(\mathrm{NIH}$, Bethesda, $\mathrm{MD})$ and normalized to the respective loading controls.

\section{Dual-luciferase reporter assay}

3'-UTR of the GRM5 or LINC00314 gene containing putative miR-129-5p targeting site was amplified by chemical synthesis and was inserted into the psiCHECK2 vector (Promega, Madison, WI, USA). When the confluence was up to $70 \%$, ADSCs were transfected with related mixtures including $50 \mathrm{ng}$ GRM5/LINC00314 wild-type or GRM5/LINC00314 mutant-type 3'-UTR reporter plasmids, miR-129-5p mimics, or miR-129-5p NC in a final concentration of $20 \mathrm{nM}$, and Lipofectamine 2000 for $48 \mathrm{~h}$. Luciferase activity was detected using the dual-luciferase reporter gene kit (Beyotime, Shanghai, China).

\section{ALP activity and staining}

ADSCs were washed with precooled PBS three times and lysed in precooled $1 \%$ Triton X-100 on ice for 30 min. Cell lysate was subjected to ALP activity determination, and the value at $405 \mathrm{~nm}$ was normalized to that of total protein concentration.

ADSCs were washed with PBS three times and fixed with $4 \%$ paraformaldehyde for $10 \mathrm{~min}$ and then added NBT-BCIP solution (BiYunTian Biotech Company, Shanghai, China) for incubation for another $15 \mathrm{~min}$. Images were captured under a microscope (Olympus DP73 Microscope, Olympus, Tokyo, Japan).

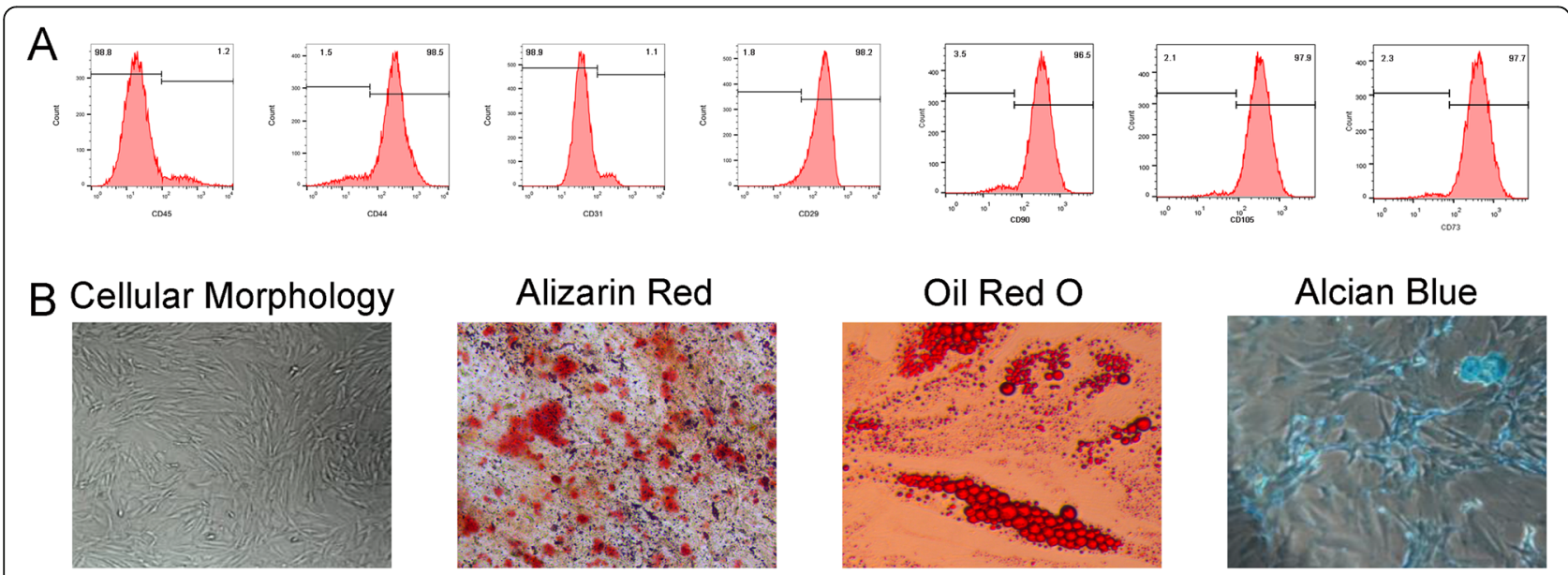

Fig. 1 Phenotype identification and trilineage differentiation potential of ADSCs. a ADSCs were negative for CD45 and CD31 and positive for CD44 and CD29. b ADSCs were induced to undergo osteogenic differentiation, adipogenic differentiation, and chondrogenic differentiation 


\section{Alizarin red staining (ARS)}

ADSCs were induced to undergo osteogenesis for 14 days. Cells were washed, fixed in 95\% ethanol for 14 min and dyed with $2 \%$ ARS-Tris-HCL solution $(\mathrm{pH}$ 4.3). Visible mineralized nodules were captured under an inverted microscope.

\section{Statistical analysis}

All data were processed by SPSS 22.0 statistical software (IBM Corp., Armonk, NY). Data are displayed as the mean \pm SD. Comparisons among multiple groups were assessed by one-way analysis of variance. The data at different time points were analyzed by repeated-measures analysis of variance. $P<0.05$ indicated statistical significance.

\section{Results}

\section{Identification of ADSCs}

To identify the phenotype of ADSCs, flow cytometry was used to detect the cell CD markers (CD44, CD29, CD31, CD45, CD73, CD90, and CD105) and trilineage differentiation potential. Flow cytometry results revealed that the ADSCs at passage 3 were negative for CD45 (1.2\%) and CD31 (1.1\%) and positive for CD44 (98.5\%), CD29 (98.2\%), CD73 (97.7\%), CD 90 (98.5\%), and CD 105 (97.8\%, Fig. 1a). In addition, ADSCs could successfully differentiate into osteoblasts (ARS, Fig. 1b),

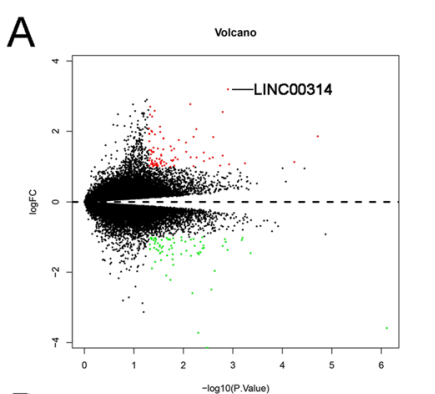

C

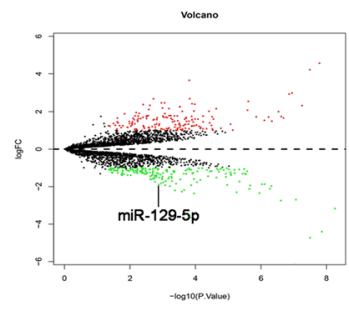

\section{B}

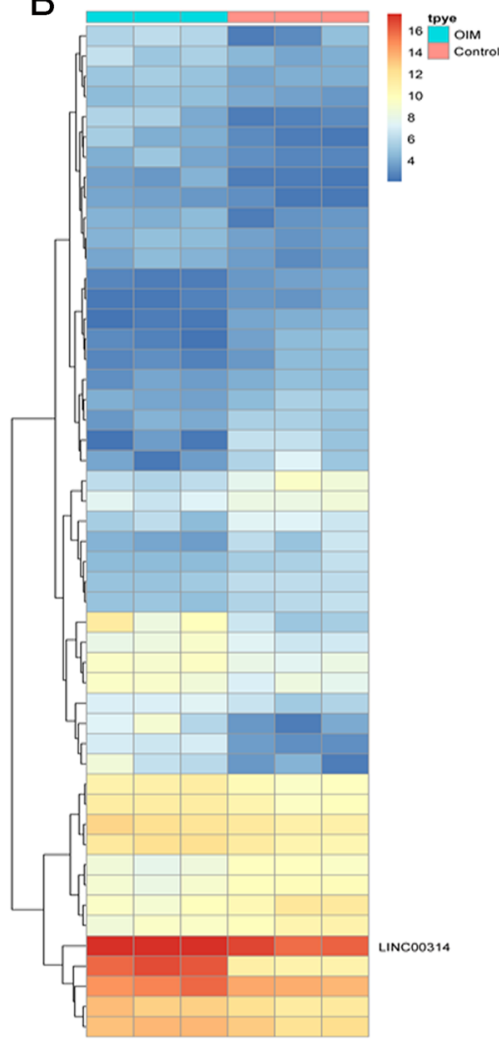

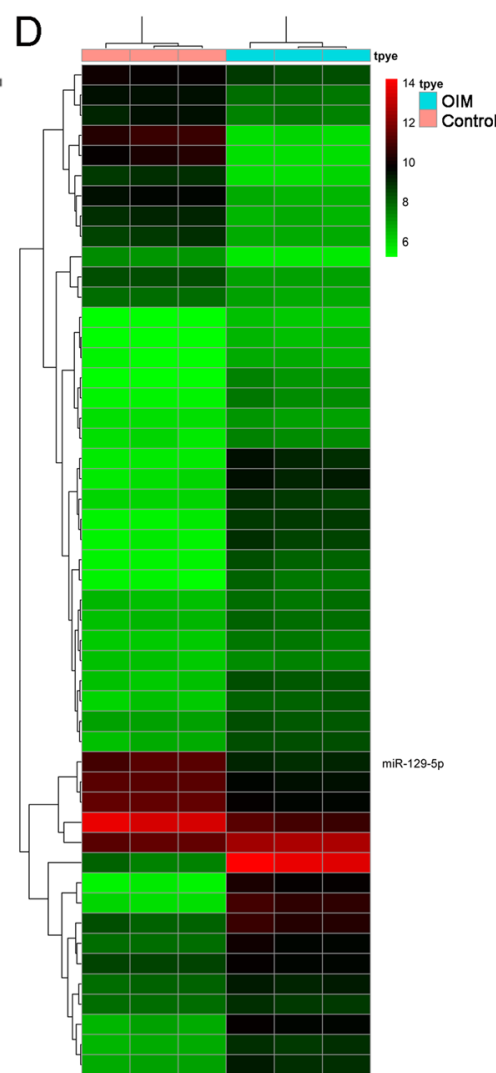

Fig. 2 The volcano plots of the significantly differentially expressed $(P<0.05$, fold change $>2)$ IncRNAs (a). Heatmap of the significantly differentially expressed IncRNAs (b). The volcano plots of the significantly differentially expressed $(P<0.05$, fold change $>2)$ microRNAs (c). Heatmap of the significantly differentially expressed microRNAs (d) 
adipocytes (Oil Red O, Fig. 1b), and chondrocytes (Alcian blue, Fig. 1b).

\section{LncRNA LINC00314 is upregulated during ADSCs osteogenic differentiation}

To further determine which lncRNA and microRNA participant into the process of ADSCs osteogenic differentiation, differentially altered lncRNA and microRNA in non-induced and induced ADSCs were analyzed further (Fig. 2a). A total of 185 upregulated lncRNAs and 153 downregulated lncRNAs were associated with osteogenic differentiation of ADSCs $(P<0.05$; $\log \mathrm{FC}>1$, Fig. 2b). Top 100 differentially expressed lncRNAs are presented in Supplementary Table S1. In particular, LINC00314 was found significantly upregulated $(\operatorname{logFC}=$ 3.19, $P=0.001)$.

In the microRNA array, a total of 105 microRNAs were identified as differentially expressed. Among these differentially expressed microRNAs, 50 were downregulated and 55 were upregulated (Fig. 2c, d). The top 100 differentially expressed miRNAs between OIM and control groups were listed in Supplementary Table S2. In particular, miR-129-5p was found significantly downregulated $(\operatorname{logFC}=-1.93, P<0.05)$.

\section{Bioinformatic analysis}

Analysis in DAVID was performed using the data profile of differentially expressed mRNAs identified by
$\mathrm{R}$ software. GO terms that included $\mathrm{BP}, \mathrm{CC}$, and $\mathrm{MF}$ are listed in Fig. 3a, b, and c, respectively. The most significantly enriched biological processes, cellular component, and molecular function terms were transcription, DNA-templated, $\mathrm{Z}$ disc and translation repressor activity, and nucleic acid binding, respectively. In the KEGG pathway analysis, the Wnt/ $\beta$-catenin signaling pathway was considered to be significantly enriched during osteogenic differentiation of ADSCs (Fig. 3d).

We then constructed a LINC00314-miRNA-target gene network using Cytoscape to visualize their interrelationships based on our miRNA-seq and target gene data (Fig. 4a). A Venn diagram was generated to assess the overlap between miRNAs targeting LINC00314, the miRNA profile, and miRNAs affecting Wnt/ $\beta$-catenin signaling, and miR-129-5p was identified (Fig. 4b). A PPI network was constructed to reveal the target gene interaction (Fig. 4c).

The network was then analyzed using the MCODE plugin, and four clustering modules were identified according to the chosen screening conditions. Clustering module 1 scored 5.2 with 5 nodes and 9 edges (Fig. 4d), clustering module 2 scored 4.8 with 11 nodes and 24 edges (Fig. 4e), clustering module 3 scored 4.2 with 4 nodes and 6 edges (Fig. 4f), and clustering module 4 scored 3.5 with 9 nodes and 15 edges (Fig. 4g).

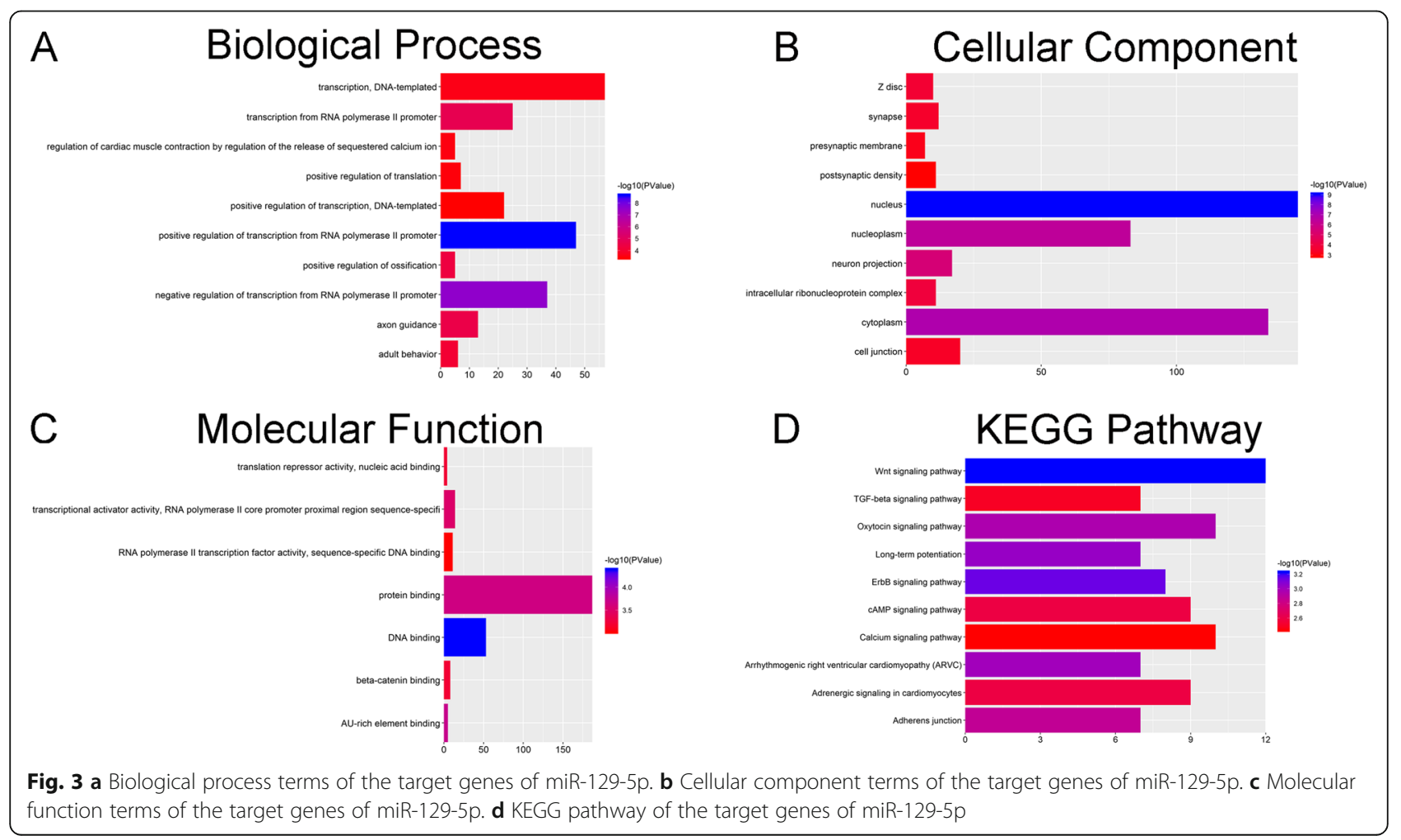



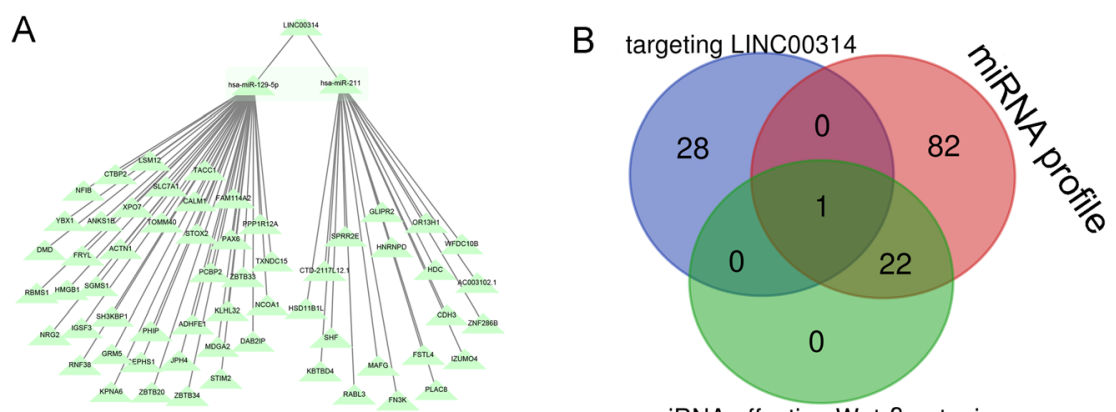

miRNA affecting Wnt- $\beta$ catenin

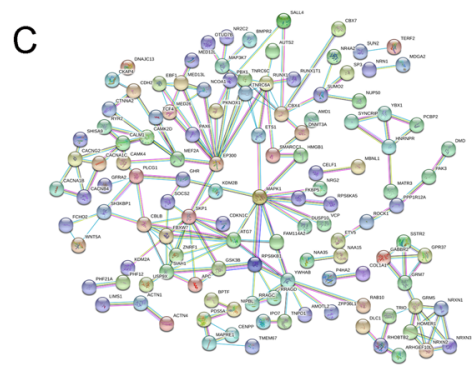

MCODE Model1
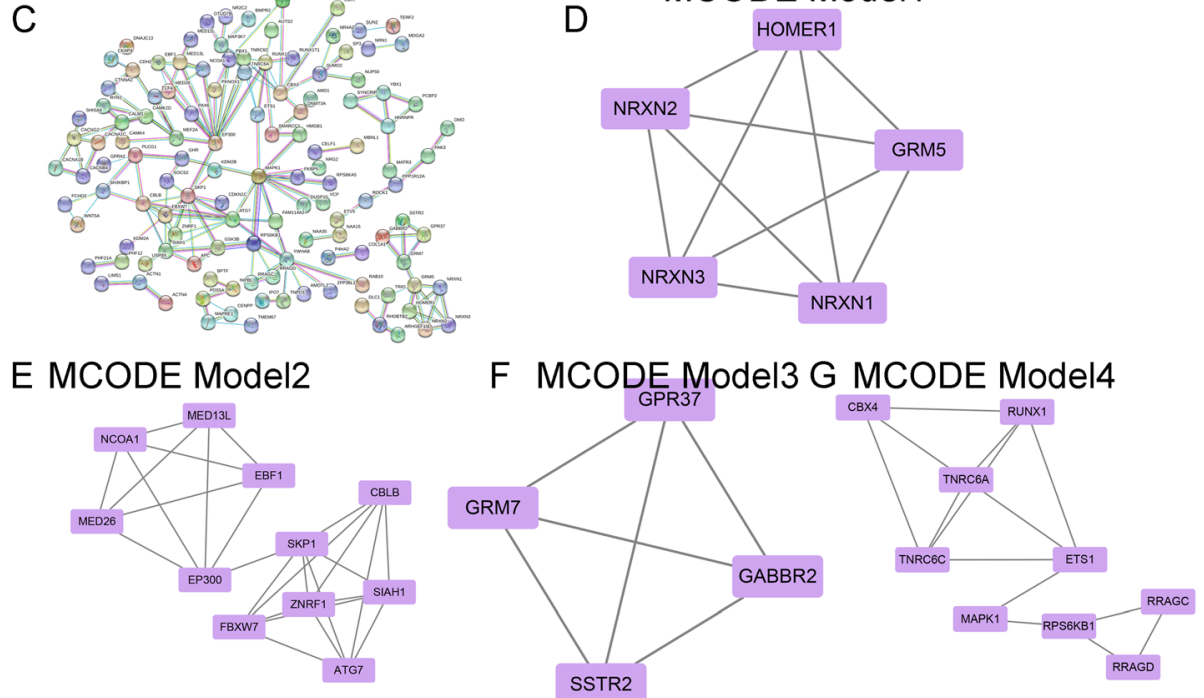

Fig. 4 a CeRNA network centered on LINC00314, miR-129-5p, and its potential target genes. b Venn diagram of miRNAs binding to LINC00314/ Wnt/ $\beta$-catenin and miRNAs from the miRNA profile identified miR-129-5p. c Protein-protein interaction of the target genes of miR-129-5p. $\mathbf{d}$ MCODE model 1, e MCODE model 2, f MCODE model 3, and $\mathbf{g}$ MCODE model 4

\section{Effects of LINC00314 on osteogenic differentiation of ADSCs}

Compared with the normal group, the induced group had significantly increased LINC00314 expression at 1,2 , and 3 weeks (Fig. 5a, $P<0.01$ ). The expression of LINC00314 was significantly increased or decreased after transfection of LINC00314 or shLINC00314, respectively, and thus the successful plasmid construction was verified (Fig. 5b). Moreover, osteogenic markers (Osterix, RUNX2, and osteocalcin) were significantly upregulated or downregulated after transfection of LINC00314 or shLINC00314, respectively (Fig. 5c-f). A large number of calcified nodules were identified by ARS and ALP activity was increased by LINC00314 overexpression, whereas these effects were suppressed by LINC00314 silencing $(P<0.05, P<0.01$, Fig. $5 \mathrm{~g})$. These data indicated that overexpression LINC00314 promoted the osteogenic differentiation of ADSCs.

\section{LINC00314 act as a sponge of miR-129-5p to stimulate ADSCs osteogenic differentiation}

The expression level of miR-129-5p exhibited a timedependent decrease during the osteogenic differentiation process $(P<0.01$, Fig. 6a). As shown in Supplementary Fig. 1, a significant negative correlation between LINC00314 and miR-129-5 was detected in induced ADSCs at 3 weeks, thus suggesting that LINC00314 may act as a sponge of miR-129-5p. Overexpression of miR-138 attenuated 3'UTRLINC00314-WT-induced luciferase activity without affecting 3'UTR- LINC00314-MUT-induced luciferase activity (Fig. 6b, c).

Compared with the NC group, agomiR-129-5p significantly increased miR-129-5p expression, while antagomiR-129-5p had the opposite effect $(P<0.01$, Fig. 6d). These experiments demonstrated the successful transfection efficiency of agomiR-129-5p and antagomiR-129-5p. The effects of increased miR-129- 

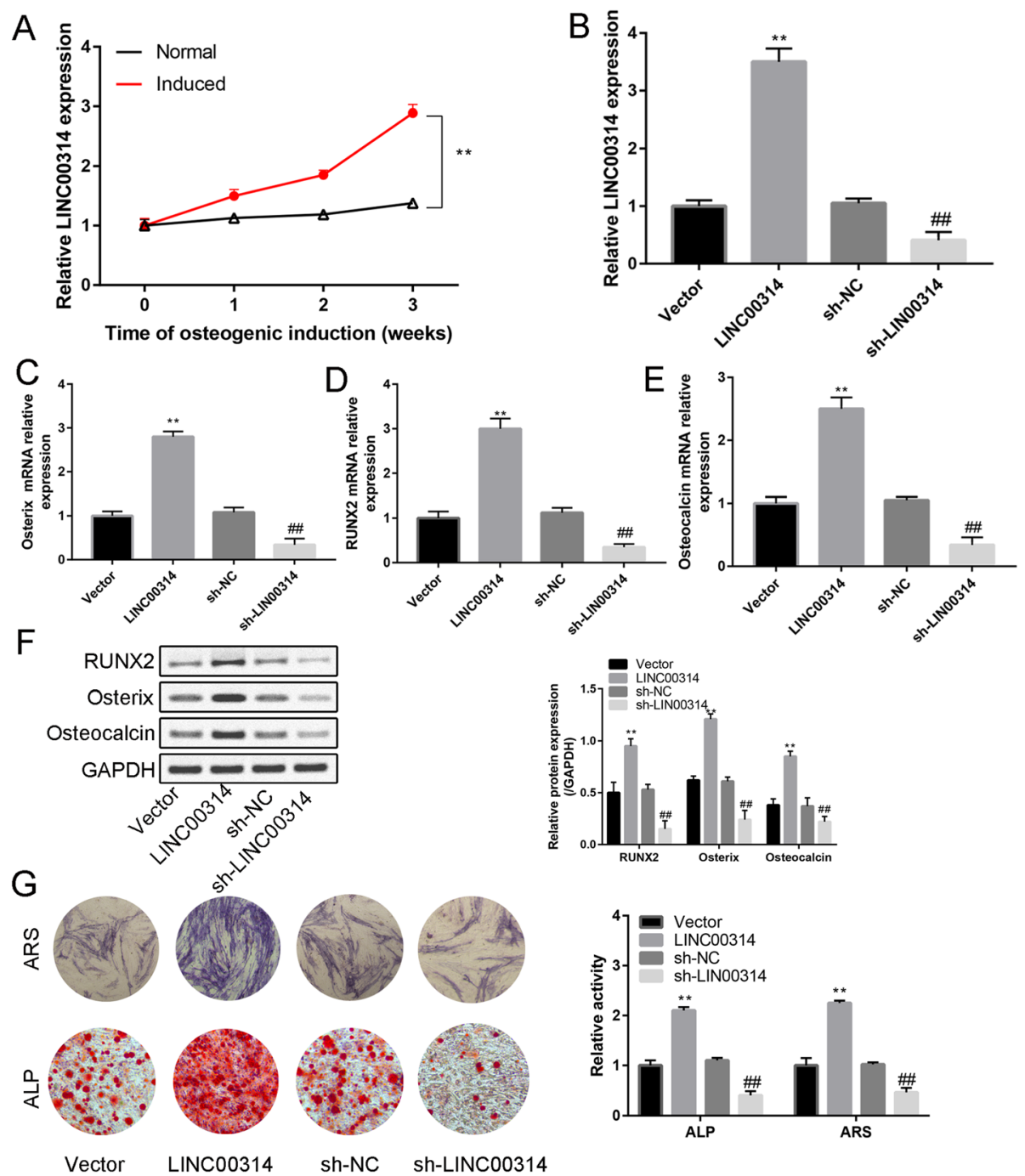

Fig. 5 a Relative expression of LINC00314 in normal and induced ADSCs from week 1 to week 3. b Relative expression of LINC00314 in induced ASCs after pcDNA3.1-LINC00314 or sh-LINC00314 transfection. Relative expression of Runx2 (c), Osterix (d), and osteocalcin (e) after transfection. $\mathbf{f}$ Western blot analysis of Runx2, Osterix, and osteocalcin after transfection. $\mathbf{g}$ Alizarin red staining (21 days) and ALP staining (14 days): changes in calcium nodule formation and ALP activity after transfection. ${ }^{*} P<0.01$, compared with the NC group; ${ }^{\# \# ~} P<0.01$, compared with the sh-NC group

$5 \mathrm{p}$ expression could be neutralized by pcDNA3.1LINC00314. The effects of inhibited miR-129-5p expression were neutralized by sh-LINC00314.

The relative expression of Runx2, Osterix, and osteocalcin in the agomiR-129-5p group was significantly downregulated compared with that in the $\mathrm{NC}$ group $(P<0.01$, Fig. 6e, f). Additionally, antagomiR129-5p significantly increased Runx2, Osterix, and osteocalcin expression compared with the $\mathrm{NC}$ group $(P<0.01$, Fig. 6e, f). This effect was partially reversible by sh-LINC00314.

ARS and ALP staining indicated that agomiR-129-5p significantly decreased calcium nodule formation and ALP activity, and LINC00314 overexpression could neutralize the agomiR-129-5p effects on osteogenic differentiation $(P<0.01$, Fig. $6 \mathrm{~g})$.

\section{MiR-129-5p regulated ASC osteogenic differentiation through GRM5}

PCR analysis showed that as the induction time increased, the relative GRM5 expression gradually increased $(P<$ $0.05)$ in the induced group compared with the normal group (Fig. 7a). There was a clear significant negative correlation between the miR-129-5p and the GRM5 expression $\left(R^{2}=0.871, P=0.001\right.$, Supplementary Fig. 2).

Moreover, compared with the corresponding NC group, agomiR-129-5p significantly inhibited GRM5 
A

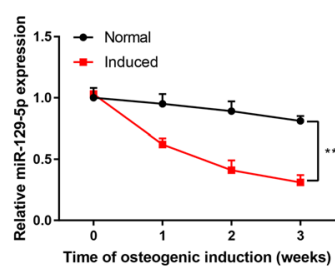

C

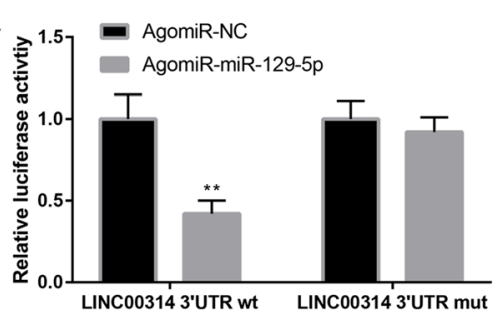

B LINC00314wt UUCCAAAAGCAAAAACAAAAAAC hsa-miR-129 CGUUCGGUCUGGCGUUUUUC

(1)
E

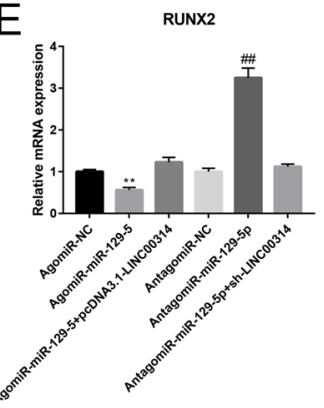

F

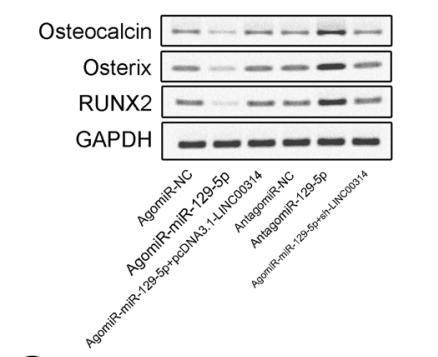

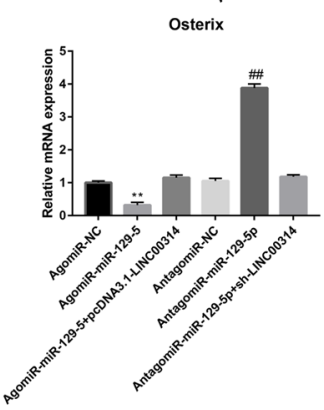

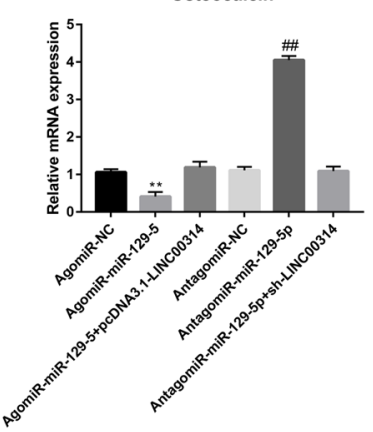

AgomiR-NC AgomiR-miR-129-5

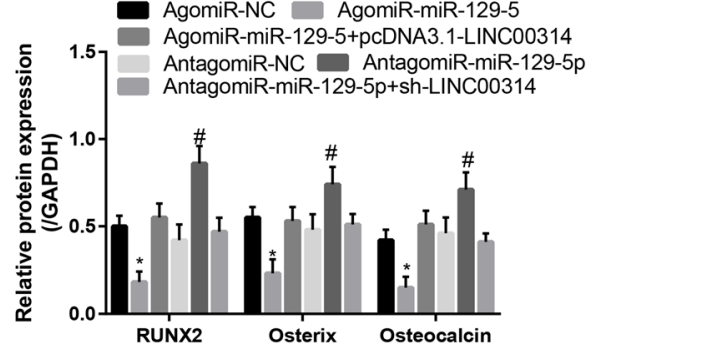

G
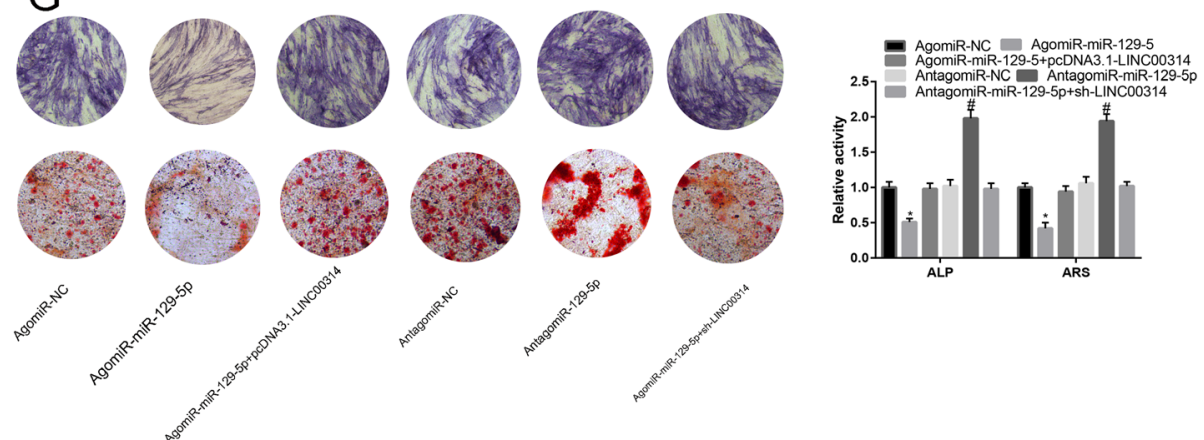

Fig. 6 a Expression levels of miR-129-5p during the osteoblast differentiation process of ADSCs, as detected by RT-PCR. b Binding site between miR-129$5 p$ and the wt/mut LINC00314 3'UTR. c Luciferase activity (LINC00314 3'UTR wt and LINC00314 3'UTR mut) in agomiR-NC- and agomiR-miR-129-5ptransfected cells. $\mathbf{d}$ Relative expression of miR-129-5p in the agomiR-NC, agomiR-miR-129-5p, agomiR-miR-129-5p+pcDNA3.1-LINC00314, antagomiR-NC, antagomiR-miR-129-5p, and antagomiR-miR-129-5p+sh-LINC00314 groups. e Relative expression of Runx2, Osterix, and osteocalcin after transfection. $\mathbf{f}$ Western blot analysis of Runx2, Osterix, and osteocalcin after transfection. $\mathbf{g}$ ARS and ALP staining in the transfection groups. ${ }^{* *} P<0.01$, compared with the NC group; ${ }^{\# \#} P<0.01$, compared with the sh-NC group 


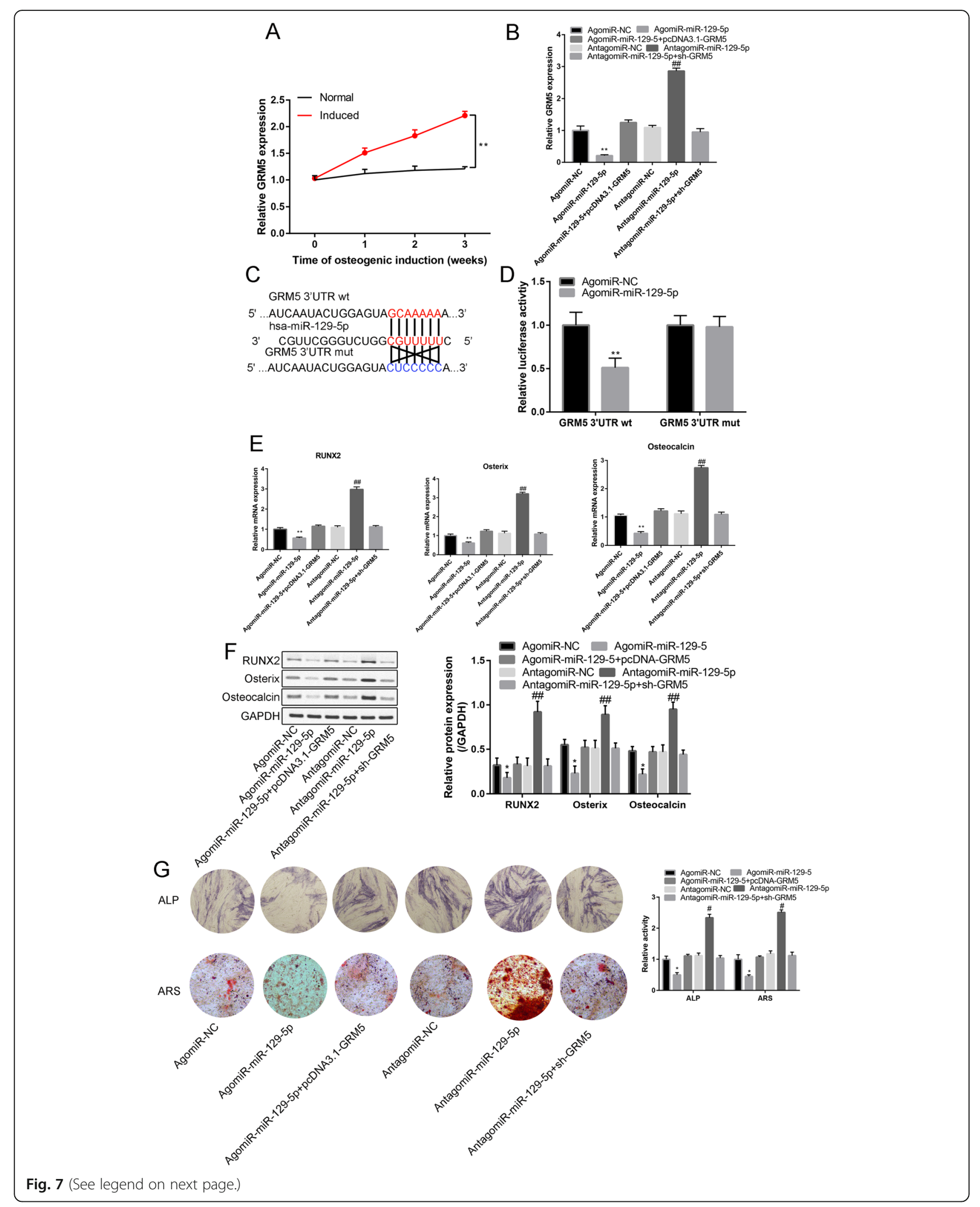


(See figure on previous page.)

Fig. 7 a Relative expression of GRM5 in the normal and induced groups from week 1 to week 3. b Relative expression of GRM5 in the agomiRNC, agomiR-miR-129-5p, agomiR-129-5p+pcDNA3.1-GEM5, antagomiR-NC, antagomiR-miR-129-5p, and antagomiR-miR-129-5p+sh-GRM5 groups. c Binding site of miR-129-5p and the wt/mut GRM5 3'UTR. $\mathbf{d}$ Luciferase activity (GRM5 3'UTR wt and GRM5 3'UTR mut) in agomiR-NC- and agomiRmiR-129-5p-transfected cells. e Relative expression of Runx2, Osterix, and osteocalcin in the agomiR-NC, agomiR-miR-129-5p, agomiR-1295p+pcDNA3.1-GEM5, antagomiR-NC, antagomiR-miR-129-5p, and antagomiR-miR-129-5p+sh-GRM5 groups. $\mathbf{f}$ Western blot analysis of Runx2, Osterix, and osteocalcin in the agomiR-NC, agomiR-miR-129-5p, agomiR-129-5p+pcDNA3.1-GEM5, antagomiR-NC, antagomiR-miR-129-5p, and antagomiR-miR-129-5p+sh-GRM5 groups. $\mathbf{g}$ ARS and ALP staining in the transfection groups. ${ }^{*} P<0.01$, compared with the NC group; ${ }^{\# \#} P<0.01$, compared with the sh-NC group

expression, while antagomiR-129-5p significantly increased GRM5 expression (Fig. 7b, $P<0.05$ ).

Figure $7 c$ presents the binding sites of miR-129-5p on GRM5 3'UTR wt. Furthermore, agomiR-129-5p significantly reduced the relative luciferase activity of the GRM5 3'UTR wt construct (Fig. 7d).

AgomiR-129-5p significantly reduced Runx2, Osterix, and osteocalcin relative expression compared with agomiR-NC (Fig. 7e, f), while the agomiR-129-5p effects on RUNX2, OOX, and OCN relative expression could be reversed by pcDNA-GRM5 and antagomiR-129-5p (Fig. 7e, f).

Figure $7 \mathrm{~g}$ shows the ARS and ALP results. Compared with the corresponding NC group, miR-129-5p significantly reduced ALP activity and calcium nodule formation. However, these inhibitory effects could be neutralized by GRM5 or antagomir-miR-129-5p (Fig. 7g)

\section{GRM5 regulated the Wnt/ $\beta$-catenin signaling pathway}

The Wnt/ $\beta$-catenin inhibitor XAV939 $(10 \mu \mathrm{M})$ was administered to identify the mechanism of action of GRM5 on the Wnt/ $\beta$-catenin signaling pathway. As illustrated in Fig. 8, we found that, compared with NC, the GRM5 inhibitor was associated with a reduction in $\mathrm{p}-\beta$-catenin and Wnt5a expression, while GRM5 mimic had the opposite effects on $\mathrm{p}-\beta$-catenin and Wnt5a expression.
Compared with the mimic group, the GRM5 mimic+XAV939 $(10 \mu \mathrm{M})$ group was associated with a reduction in $\mathrm{p}-\beta$-catenin and Wnt5a expression. These findings suggested that GRM5 regulated the $\mathrm{Wnt} / \beta$-catenin signaling pathway and thus promoted osteogenic differentiation of ADSCs.

\section{Discussion}

We firstly identified LINC00314 plays a critical role in the regulation of osteogenic differentiation of ADSCs. Gain and loss of LINC00314/miR-129-5p/GRM5 function studies revealed that LINC00314 could positively regulate the early and late osteogenesis of ADSCs. Furthermore, the LINC00314/miR-129-5p/GRM5 axis exerts its osteogenic effects on ADSCs through the Wnt/ $\mathrm{B}$ catenin signaling pathway.

Recently, ADSCs have gained considerable attention in tissue-engineered bone due to the fat tissue is a rich source of MSCs with minimal injury and less painful [20]. Previous studies have demonstrated that ADSCs can trigger differentiation into adipocytes, chondrocytes, and osteoblasts under specific optimal medium condition [21-23]. Therefore, the promotion of osteogenic differentiation of ADSCs played an important role.

In this study, microarray was firstly conducted to reveal the differentially expressed lncRNAs and identified LINC00314 as the most statistically significant lncRNA.

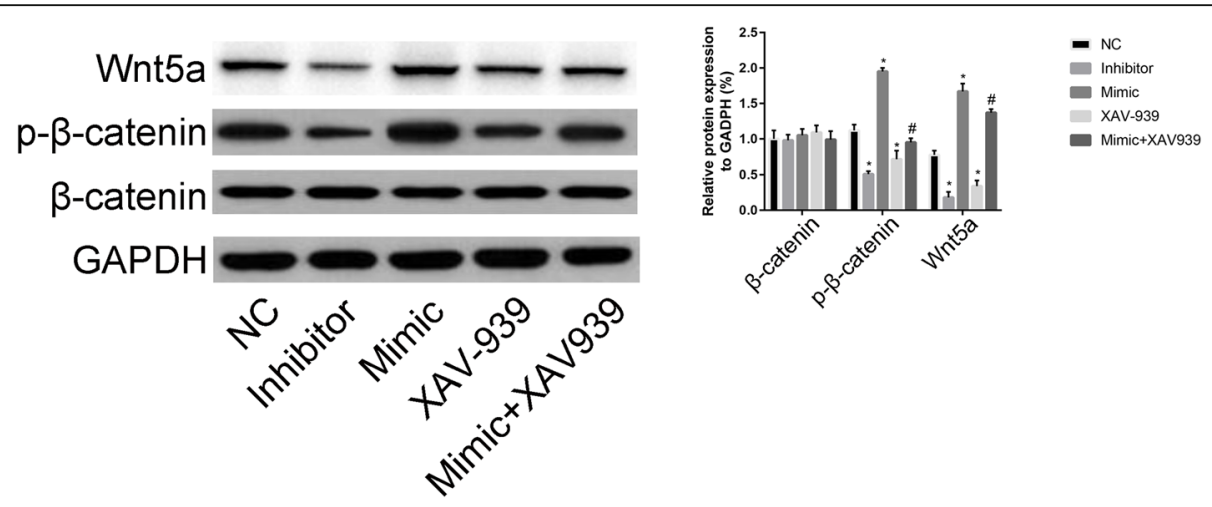

Fig. 8 Effects of the miR-129-5p/GRM5 axis on the Wnt/ $\beta$-catenin signaling pathway. Effects of GRM5 mimic, GRM5 inhibitor, XAV-939, and GRM5

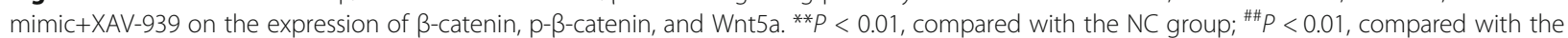
sh-NC group 


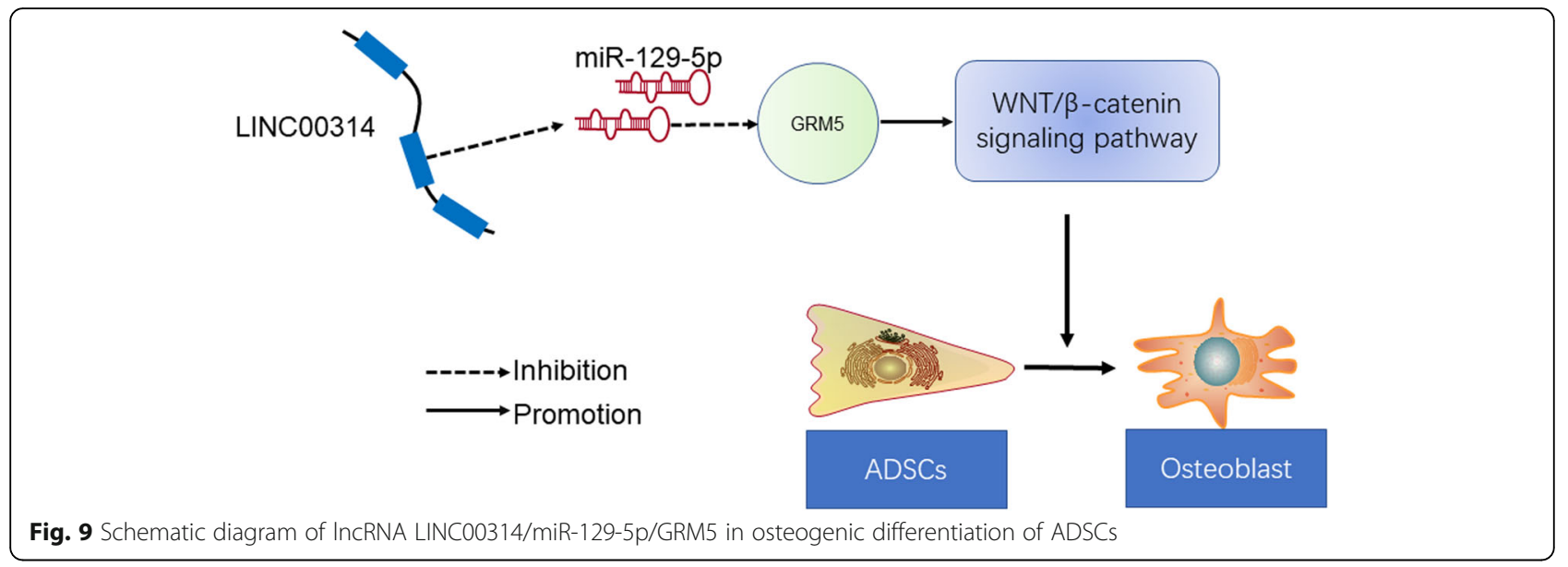

Yi et al. [24] revealed that IncRNA MALAT1 promoted osteogenic differentiation of ADSCs by directly targeting with miR-30. Wu et al. [10] also found that IncRNA HIF1A-AS2 acts as a sponge of miR-665 to regulate IL-6 expression and facilitates bone formation through regulating PI3K/Akt signaling pathway.

We predicted the LINC00314 binding site by using the bioinformatics databases (miRcode). The overlap between the predicted microRNAs and microRNA array results found that miR-129-5p was linked with both LINC00314 and GRM5. Previous studies showed that miR-129-5p function as oncogenes or tumor suppressors during the occurrence and development of multiple cancers, including retinoblastoma [13], non-small cell lung cancer [25], and hepatocellular carcinoma [26]. Its functional role in osteogenic differentiation of ADSCs is poorly demonstrated.

We found that overexpressing miR-129-5p could significantly downregulate ALP activity and calcium deposits. Thus, we concluded that LINC00314 is a sponge of miR-129-5p. Han et al. [27] found that miR-129-5p directly targeting ROCK1 and suppresses osteosarcoma progression, which is consistent with our research findings. Luciferase assays showed that miR-129-5p directly bind to the 3'UTR of GRM5. Finally, GRM 5 could regulate the Wnt/ $\beta$-catenin signaling pathway to induce the osteogenic differentiation of ADSCs. Wnt $/ \beta$-catenin is a very complex signaling pathway and is involved in ADSC differentiation [28]. Xu et al. [29] found miR-889 directly targeting WNT7A and subsequently suppressing bone formation.

The Wnt/ $\beta$-catenin signaling pathway is closely related to osteoblast differentiation of ADSCs. Anabolic agents that stimulate the $\mathrm{Wnt} / \beta$-catenin signaling pathway can be used to treat osteoblast-related diseases such as osteoporosis. Recently, antisclerostin and anti-dickkopf antibodies were used to conduct the preliminary clinical trial and show great potential for the treatment of osteoporosis [30-32]. Western blotting and loss/gain-of-function assays of the Wnt pathway confirmed that upregulated expression of GRM5 induced the activity of the Wnt signaling pathway, which promoted the osteogenic differentiation of ADSCs.

\section{Conclusions}

In conclusion, we propose that the LINC00314/miR129-5p/GRM5 axis positively regulates the early and late osteogenic differentiation ability by modulating the Wnt/ $\beta$-catenin signaling pathway in ADSCs (Fig. 9). LINC00314 was upregulated during osteogenic differentiation of ADSCs, which enhanced its miR-129-5p sponge function, thereby promoting GRM5 expression by activating the Wnt/ $\beta$-catenin signaling pathway. Our findings could provide a new target for controlling osteogenesis in ADSCs, which are crucial to bone tissue engineering and treatment for bone diseases.

\section{Supplementary information}

Supplementary information accompanies this paper at https://doi.org/10. 1186/s13287-020-01754-Z.

Additional file 1: Supplement Figure 1. Relationship between LINC00314 and miR-129-5p in ADSCs after osteogenic differentiation for 3 weeks

Additional file 2: Supplement Figure 2. Relationship between miR129-5p and GRM5 in ADSCs after osteogenic differentiation for 3 weeks.

Additional file 3: Supplementary Table S1. Differentially expressed IncRNAs between induced and non-induced ADSCs. logFC: log fold change; AveExpr: average expression; adj. P.Val: adjustive $P$ value.

Additional file 4: Supplementary Table S2. Differentially expressed miRNAs between induced and non-induced ADSCs. logFC: log fold change; AveExpr: average expression; adj. P.Val: adjustive $P$ value.

\section{Abbreviations}

ADSCs: Adipose-derived stem cells; IncRNAs: Long noncoding RNAs; ARS: Alizarin red staining; ALP: Alkaline phosphatase; BMD: Bone mineral density; BMSCs: Bone marrow mesenchymal stem cells; miRNA: microRNA; FBS: Fetal bovine serum; DMEM: Dulbecco's modified Eagle medium; 
OIM: Osteogenic induction medium; BSA: Bovine serum albumin; DAVID: Database for Annotation, Visualization and Integrated Discovery; GO: Gene ontology; KEGG: Kyoto Encyclopedia of Genes and Genomes; BP: Biological process; CC: Cellular component; MF: Molecular function; STRING: Search Tool for the Retrieval of Interacting Genes; PPI: Proteinprotein interaction; cDNA: Complementary deoxyribose nucleic acid; PBS: Phosphate-buffered saline

\section{Acknowledgements}

Not applicable.

\section{Authors' contributions}

ZLS and HZ conducted the experiment. ZYF and WM performed the statistical analysis. YZS and ML re-checked the data and wrote the draft. TQL and SXC revised the manuscript. ZLS and GJF guided the experiment and helped the first author perform the experiment. The author(s) read and approved the final manuscript.

\section{Funding}

Not applicable.

\section{Availability of data and materials}

The datasets used and analyzed during the current study are available from the corresponding author on reasonable request.

\section{Ethics approval and consent to participate}

All experiments have been authorized by the ethics committee of the Second Hospital of Hebei Medical University. Informed consent was collected from all participants involved in this study.

\section{Consent for publication}

Not applicable.

\section{Competing interests}

All of the authors listed in this manuscript declare no competing interests.

Received: 12 April 2020 Revised: 28 May 2020

Accepted: 1 June 2020 Published online: 17 June 2020

\section{References}

1. An J, Yang H, Zhang Q, Liu C, Zhao J, Zhang L, Chen B. Natural products for treatment of osteoporosis: the effects and mechanisms on promoting osteoblast-mediated bone formation. Life Sci. 2016:147:46-58.

2. Song N, Zhao Z, Ma X, Sun X, Ma J, Li F, Sun L, LV J. Naringin promotes fracture healing through stimulation of angiogenesis by regulating the VEGFNEGFR-2 signaling pathway in osteoporotic rats. Chem Biol Interact. 2017;261:11-7

3. Cauley JA. Osteoporosis: fracture epidemiology update 2016. Curr Opin Rheumatol. 2017:29:150-6.

4. Jin Z, Chen J, Shu B, Xiao Y, Tang D. Bone mesenchymal stem cell therapy for ovariectomized osteoporotic rats: a systematic review and meta-analysis. BMC Musculoskelet Disord. 2019:20:556.

5. Su X, Liao L, Shuai Y, Jing H, Liu S, Zhou H, Liu Y, Jin Y. MiR-26a functions oppositely in osteogenic differentiation of BMSCs and ADSCs depending on distinct activation and roles of Wnt and BMP signaling pathway. Cell Death Dis. 2015;6:e1851.

6. Chen S, Zhu J, Wang M, Huang Y, Qiu Z, Li J, Chen X, Chen H, Xu M, Liu J, She $M$, Li H, Yang X, Wang Y, Cai X. Comparison of the therapeutic effects of adiposederived and bone marrow mesenchymal stem cells on erectile dysfunction in diabetic rats. Int J Mol Med. 2019;44:1006-14.

7. Wu Y, Jiang Y, Liu Q, Liu CZ. IncRNA H19 promotes matrix mineralization through up-regulating IGF1 by sponging miR-185-5p in osteoblasts. BMC Mol Cell Biol. 2019:20:48

8. Zhang Y, Cao X, Li P, Fan Y, Zhang L, Ma X, Sun R, Liu Y, Li W. LncRNA NKILA integrates RXFP1/AKT and NF-kappaB signalling to regulate osteogenesis of mesenchymal stem cells. J Cell Mol Med. 2019. https://doi. org/10.1111/jcmm.14759.

9. Fu L, Peng S, Wu W, Ouyang Y, Tan D, Fu X. LncRNA HOTAIRM1 promotes osteogenesis by controlling JNK/AP-1 signalling-mediated RUNX2 expression. J Cell Mol Med. 2019;23:7517-24.
10. Wu R, Ruan J, Sun Y, Liu M, Sha Z, Fan C. Long non-coding RNA HIF1A-AS2 facilitates adipose-derived stem cells (ASCs) osteogenic differentiation through miR-665/IL6 axis via PI3K/Akt signaling pathway. Stem Cell Res Ther. 2018;9:348.

11. Liu X, Zhu W, Wang L, Wu J, Ding F, Song Y. miR-145-5p suppresses osteogenic differentiation of adipose-derived stem cells by targeting semaphorin 3A. In Vitro Cell Dev Biol Anim. 2019;55:189-202.

12. Li J, Hu C, Han L, Liu L, Jing W, Tang W, Tian W, Long J. MiR-154-5p regulates osteogenic differentiation of adipose-derived mesenchymal stem cells under tensile stress through the Wnt/PCP pathway by targeting Wnt11. Bone. 2015:78:130-41.

13. Liu Y, Liang G, Wang H, Liu Z. MicroRNA-129-5p suppresses proliferation, migration and invasion of retinoblastoma cells through PI3K/AKT signaling pathway by targeting PAX6. Pathol Res Pract. 2019;215:152641.

14. Yang R, Cai X, Li J, Liu F, Sun T. Protective effects of MiR-129-5p on acute spinal cord injury rats. Med Sci Monit. 2019;25:8281-8.

15. Valenti MT, Deiana M. Physical exercise modulates miR-21-5p, miR-129-5p, miR-378-5p, and miR-188-5p expression in progenitor cells promoting osteogenesis. Cells. 2019:8:E742.

16. Luo WY, Xing SQ, Zhu P, Zhang CG, Yang HM, Van Halm-Lutterodt N, Gu L, Zhang H. PDZ scaffold protein CAL couples with metabotropic glutamate receptor 5 to protect against cell apoptosis and is a potential target in the treatment of Parkinson's disease. Neurotherapeutics. 2019;16:761-83.

17. Zuk PA, Zhu M, Mizuno H, Huang J, Futrell JW, Katz AJ, Benhaim P, Lorenz $\mathrm{HP}$, Hedrick MH. Multilineage cells from human adipose tissue: implications for cell-based therapies. Tissue Eng. 2001;7:211-28.

18. Zhu HR, Yu XN, Zhang GC, Shi X, Bilegsaikhan E, Guo HY, Liu LL, Cai Y, Song GQ, Liu TT, Dong L, Janssen HLA, Weng SQ, Wu J, Shen XZ, Zhu JM. Comprehensive analysis of long noncoding RNAmessenger RNAmicroRNA coexpression network identifies cell cyclerelated IncRNA in hepatocellular carcinoma. Int J Mol Med. 2019;44:1844-54.

19. Jiang B, Xiang Z, Ai Z, Wang H, Li Y, Ji W, Li T. Generation of cardiac spheres from primate pluripotent stem cells in a small molecule-based 3D system. Biomaterials. 2015:65:103-14

20. Yang $Q$, Teng BH, Wang LN, Li K, Xu C, Ma XL, Zhang Y, Kong DL, Wang LY, Zhao YH. Silk fibroin/cartilage extracellular matrix scaffolds with sequential delivery of TGF-beta3 for chondrogenic differentiation of adipose-derived stem cells. Int J Nanomedicine. 2017;12:6721-33.

21. Correa D, Lietman SA. Articular cartilage repair: current needs, methods and research directions. Semin Cell Dev Biol. 2017:62:67-77.

22. Panadero JA, Lanceros-Mendez S, Ribelles JL. Differentiation of mesenchymal stem cells for cartilage tissue engineering: individual and synergetic effects of three-dimensional environment and mechanical loading. Acta Biomater. 2016;33:1-12

23. Zeng G, Lai K, Li J, Zou Y, Huang H, Liang J, Tang X, Wei J, Zhang P. A rapid and efficient method for primary culture of human adipose-derived stem cells. Organogenesis. 2013:9:287-95.

24. Yi J, Liu D, Xiao J. LnCRNA MALAT1 sponges miR-30 to promote osteoblast differentiation of adipose-derived mesenchymal stem cells by promotion of Runx2 expression. Cell Tissue Res. 2019;376:113-21.

25. Li G, Xie J, Wang J. Tumor suppressor function of miR-129-5p in lung cancer. Oncol Lett. 2019;17:5777-83.

26. Shaker OG, Abdelwahed MY, Ahmed NA, Hassan EA, Ahmed TI, Abousarie MA, Ayoub SE. Evaluation of serum long noncoding RNA NEAT and MiR129-5p in hepatocellular carcinoma. IUBMB Life. 2019:71:1571-8.

27. Han C, Wang W. MicroRNA-129-5p suppresses cell proliferation, migration and invasion via targeting ROCK1 in osteosarcoma. Mol Med Rep. 2018;17: 4777-84.

28. Ai G, Meng M, Wang L, Shao X, Li Y, Cheng J, Tong X, Cheng Z. microRNA196a promotes osteogenic differentiation and inhibit adipogenic differentiation of adipose stem cells via regulating beta-catenin pathway. Am J Transl Res. 2019:11:3081-91.

29. Xu G, Ding Z, Shi HF. The mechanism of miR-889 regulates osteogenesis in human bone marrow mesenchymal stem cells. J Orthop Surg Res. 2019:14: 366.

30. Li X, Ominsky MS, Warmington KS, Morony S, Gong J, Cao J, Gao Y, Shalhoub V, Tipton B, Haldankar R, Chen Q, Winters A, Boone T, Geng Z, Niu QT, Ke HZ, Kostenuik PJ, Simonet WS, Lacey DL, Paszty C. Sclerostin antibody treatment increases bone formation, bone mass, and bone strength in a rat model of postmenopausal osteoporosis. J Bone Miner Res. 2009;24:578-88. 
31. Padhi D, Jang G, Stouch B, Fang L, Posvar E. Single-dose, placebocontrolled, randomized study of AMG 785, a sclerostin monoclonal antibody. J Bone Miner Res. 2011;26:19-26.

32. Glantschnig H, Scott $K$, Hampton R, Wei N, McCracken P, Nantermet P, Zhao JZ, Vitelli S, Huang L, Haytko P, Lu P, Fisher JE, Sandhu P, Cook J, Williams D, Strohl W, Flores O, Kimmel D, Wang F, An Z. A rate-limiting role for

Dickkopf-1 in bone formation and the remediation of bone loss in mouse and primate models of postmenopausal osteoporosis by an experimental therapeutic antibody. J Pharmacol Exp Ther. 2011;338:568-78.

\section{Publisher's Note}

Springer Nature remains neutral with regard to jurisdictional claims in published maps and institutional affiliations.

Ready to submit your research? Choose BMC and benefit from:

- fast, convenient online submission

- thorough peer review by experienced researchers in your field

- rapid publication on acceptance

- support for research data, including large and complex data types

- gold Open Access which fosters wider collaboration and increased citations

- maximum visibility for your research: over $100 \mathrm{M}$ website views per year

At $\mathrm{BMC}$, research is always in progress.

Learn more biomedcentral.com/submissions 